\title{
ON A SIMILARITY INVARIANT FOR COMPACT OPERATORS
}

\author{
BY \\ REESE T. PROSSER( $\left.{ }^{(}\right)$
}

Dedicated to the Memory of Hazleton Mirkil

Let $\mathscr{H}$ be a Hilbert space, and $\mathscr{K}$ the algebra of all compact operators acting on $\mathscr{H}$. If $K \in \mathscr{K}$, then $K=W A$, where $A=\left(K^{*} K\right)^{1 / 2}$ is compact and positive, and $W$ is a partial isometry mapping the range of $A$ isometrically onto the range of $K$. If $k_{n}$ and $a_{n}$ are the $n$th eigenvalues, counted with multiplicities and arranged in order of decreasing magnitude, of $K$ and $A$, respectively, then $0 \leqq\left|k_{n}\right| \leqq a_{n}$, and $a_{n} \downarrow 0$ as $n \uparrow \infty$.

For each $K \in \mathscr{K}$ and $p, 0<p \leqq \infty$, put

$$
\begin{aligned}
\|K\|_{p}=\|A\|_{p} & =\left(\sum_{n=1}^{\infty} a_{n}^{p}\right)^{1 / p}, & & 0<p<\infty, \\
& =\sup \left\{a_{n}: 1 \leqq n<\infty\right\}, & & p=\infty .
\end{aligned}
$$

Then $0 \leqq\|K\|_{p} \leqq \infty$, and $\|K\|_{p} \downarrow$ as $p \uparrow$. Moreover,

Lemma 1. If $K, M \in \mathscr{K}$ and $B, C$ are bounded operators on $\mathscr{H}$, then

$$
\begin{array}{cc}
\|K+M\|_{p} \leqq 2^{1 / p}\left\{\|K\|_{p}^{p}+\|M\|_{p}^{p}\right\}^{1 / p}, & 0<p \leqq 1, \\
\leqq 2^{1 / p}\left\{\|K\|_{p}+\|M\|_{p}\right\}, & 1 \leqq p \leqq \infty, \\
\|K M\|_{p} \leqq 2^{1 / p}\|K\|_{r}\|M\|_{s}, & \text { where } 1 / p=1 / r+1 / s, \\
\|B K C\|_{p} \leqq\|B\|\|K\|_{p}\|C\|, & \\
\left\|K^{*}\right\|_{p}=\|K\|_{p} . &
\end{array}
$$

Proof. See [2, Lemma 9, p. 1093].

Now for each $K \in \mathscr{K}$, put

$$
\tau(K)=\operatorname{glb}\left\{p:\|K\|_{p}<\infty\right\}=\operatorname{glb}\left\{p: A^{p} \in \text { trace class }\right\} .
$$

Then $0 \leqq \tau(K) \leqq \infty$, and from Lemma 1 we have

Received by the editors June 23, 1967.

(1) The results obtained here were suggested primarily by the work of R. M. Dudley [1], preprints of which we gratefully acknowledge. 
Corollary 2. If $K, M \in \mathscr{K}$ and $B, C$ are bounded operators on $\mathscr{H}$, then

$$
\begin{aligned}
\tau(K+M) & \leqq \max \{\tau(K), \tau(M)\}, \\
\tau(K M) & \leqq \tau(K) \tau(M) /(\tau(K)+\tau(M)), \\
\tau(B K C) & \leqq \tau(K) \\
\tau\left(K^{*}\right) & =\tau(K) .
\end{aligned}
$$

Proof. See Lemma 1.

In particular, it follows from (9) that if $B$ is a bounded invertible operator, then

$$
\tau\left(B K B^{-1}\right)=\tau(K) \text {. }
$$

Hence $\tau$ is a similarity invariant for the class $\mathscr{K}$ of compact operators. It is clear from the definitions that if $K$ is of finite rank, trace class, or Hilbert-Schmidt class, then $\tau(K)=0$, $\leqq 1$, or $\leqq 2$, respectively. Moreover, we have

LEMMA 3. If $K, M \in \mathscr{K}$, and if, for all $\phi \in \mathscr{H}$,

$$
\|K \phi\| \leqq \text { const }\|M \phi\|,
$$

then $\tau(K) \leqq \tau(M)$.

Proof. If $\|K \phi\|^{2}=\left(K^{*} K \phi, \phi\right) \leqq$ const $\|M \phi\|^{2}=\left(M^{*} M \phi, \phi\right)$ for all $\phi \in \mathscr{H}$, then $K^{*} K \leqq$ const $M^{*} M$. If $a_{n}$ and $b_{n}$ are the $n$th eigenvalues of $\left(K^{*} K\right)^{1 / 2}$ and $\left(M^{*} M\right)^{1 / 2}$, counted with multiplicities and arranged in order of decreasing magnitude, then it follows that $a_{n} \leqq$ const $b_{n}\left[2\right.$, p. 909]. Hence if $\sum b_{n}^{p}<\infty$, for any $p, 0<p<\infty$, then $\sum a_{n}^{p}<\infty$, and $\tau(K) \leqq \tau(M)$.

Thus $\tau(K)$ provides a measure of the "size" of $K$. In this paper we propose to explore this idea by introducing various other measures of the "size" of $K$ and relating them to $\tau(K)$.

All of our measures of the "size" of $K$ are given in terms of the asymptotic behavior of certain positive sequences or functions associated with $K$. If $\left\{b_{n}\right\}$ and $\left\{c_{n}\right\}$ are arbitrary monotone-increasing positive sequences, with $b_{n}, c_{n} \uparrow \infty$ as $n \uparrow \infty$, then the asymptotic behavior of $b_{n}$ may be compared with that of $c_{n}$ by introducing the relative order of growth $\gamma$, defined by the formula

$$
\begin{aligned}
\gamma & =\operatorname{glb}\left\{\mu>0: b_{n} \leqq \text { const } c_{n}^{\mu}\right\} \\
& =\infty \text { if no such } \mu>0 \text { exists. }
\end{aligned}
$$

Then clearly $0 \leqq \gamma \leqq \infty$, and if $0<\gamma-\varepsilon<\gamma<\gamma+\varepsilon<\infty$, we have $b_{n} \leqq$ const $c_{n}^{\gamma+\varepsilon}$ for all $n$, and $b_{n} \geqq$ const $c_{n}^{\gamma-\varepsilon}$ for arbitrarily large $n$. The computation of the relative order of growth is facilitated by the formula

$$
\gamma=\limsup _{n \rightarrow \infty} \frac{\log b_{n}}{\log c_{n}}
$$


Similarly, if $\left\{b_{n}\right\}$ and $\left\{c_{n}\right\}$ are monotone-decreasing sequences, with $b_{n}, c_{n} \downarrow 0$ as $n \downarrow \infty$, then we introduce the relative order of decay $\delta$, defined by the formula

$$
\begin{aligned}
\delta & =\operatorname{lub}\left\{\mu>0: b_{n} \leqq \text { const } c_{n}^{\mu}\right\}, \\
& =\infty \text { if no such } \mu>0 \text { exists. }
\end{aligned}
$$

Then $0 \leqq \delta \leqq \infty$, and if $0<\delta-\varepsilon<\delta<\delta+\varepsilon<\infty$, we have $b_{n} \leqq$ const $c_{n}^{\delta-\varepsilon}$ for all $n$, and $b_{n} \geqq$ const $c_{n}^{\delta+\varepsilon}$ for arbitrarily large $n$. The computation of $\delta$ is given by

$$
\delta=\liminf _{n \rightarrow \infty} \frac{\log b_{n}}{\log c_{n}}
$$

When $c_{n}=n$ (or $1 / n$ ) we call $\gamma$ (or $\delta$ ) simply the order of growth (or order of decay, respectively) of $b_{n}$.

From now on let $K \in \mathscr{K}$ be a fixed compact operator acting on $\mathscr{H}$, and $A$ $=\left(K^{*} K\right)^{1 / 2}$. Let $\mathscr{B}$ be the unit ball in $\mathscr{H}$, and $\mathscr{E}$ the (compact, convex, symmetric) image of $\mathscr{B}$ under $K$. The "size" of $K$ is reflected in the "size" of $\mathscr{E}$, which can be measured in several different ways. Among them we cite the following:

Definition 4 (The Metric Volume [1]). Let $\mathscr{E}$ be any compact convex

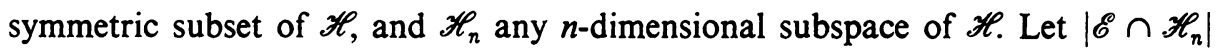
denote the $n$-dimensional Lebesgue volume of $\mathscr{E} \cap \mathscr{H}_{n}$, and put $V_{n}=\sup \left|\mathscr{E} \cap \mathscr{H}_{n}\right|$, the supremum taken over all the $\mathscr{H}_{n}$ in $\mathscr{H}$. Thus $V_{n}$ is the least upper bound of the volumes of the $n$-dimensional sections of $\mathscr{E}$, and is called the $n$-dimensional metric volume of $\mathscr{E}$.

Since $\mathscr{E}$ is compact, $V_{n} \downarrow 0$ as $n \uparrow \infty$. The rate of decrease of $V_{n}$ can be effectively compared with that of the volume $B_{n}$ of the unit $n$-ball $\mathscr{B}_{n}=\mathscr{B} \cap \mathscr{H}_{n}$. Put

$$
\begin{aligned}
\beta(\mathscr{E}) & =\operatorname{lub}\left\{\mu>0: V_{n} \leqq \text { const }\left(B_{n}\right)^{\mu}\right\}, \\
& =0 \text { if no such } \mu>0 \text { exists. }
\end{aligned}
$$

Then $\beta(\mathscr{E})$ is the order of decay of the metric volume of $\mathscr{E}$ relative to that of the unit ball $\mathscr{B}$. When $\mathscr{E}=K(\mathscr{B})$, we shall write $\beta(\mathscr{E})=\beta(K)$.

Definition 5 (The Metric Width [5]). Let $\mathscr{E}$ be any compact convex symmetric subset of $\mathscr{H}$, and $\mathscr{H}_{n}$ any $n$-dimensional subspace of $\mathscr{H}_{\text {. Let }} d\left(\mathscr{E}, \mathscr{H}_{n}\right)$ denote the maximal orthogonal distance from $\mathscr{E}$ to $\mathscr{H}_{n}$, and put $w_{n}=\inf d\left(\mathscr{E}, \mathscr{H}_{n}\right)$, the infinum taken over all the $\mathscr{H}_{n}$ in $\mathscr{H}$. Then $w_{n}$ is the greatest lower bound of the distance from $\mathscr{E}$ to the $n$-dimensional subspaces of $\mathscr{H}$, and is called the $n$-dimensional metric width of $\mathscr{E}$.

Since $\mathscr{E}$ is compact, $w_{n} \downarrow 0$ as $n \uparrow \infty$. The rate of decrease of $w_{n}$ can be effectively compared with that of the sequence $1 / n$. Put

$$
\begin{aligned}
\omega(\mathscr{E}) & =\operatorname{lub}\left\{\mu>0: w_{n} \leqq \text { const }(1 / n)^{\mu}\right\}, \\
& =0 \text { if no such } \mu>0 \text { exists. }
\end{aligned}
$$

Then $\omega(\mathscr{E})$ is the order of decay of the metric width of $\mathscr{E}$ (relative to $1 / n$ ). When $\mathscr{E}=K(\mathscr{B})$, we write $\omega(\mathscr{E})=\omega(K)$. 
Definition 6 (The Metric Entropy [5]). Let $\mathscr{E}$ be any compact convex symmetric subset of $\mathscr{H}$, and $\varepsilon>0$. Let $\mathscr{U}(\varepsilon)$ be any finite covering of $\mathscr{E}$ by open balls of radius $\varepsilon$, and let card $\mathscr{U}(\varepsilon)$ denote the number of balls in $\mathscr{U}(\varepsilon)$. Put $N(\varepsilon)=$ inf card $\mathscr{U}(\varepsilon)$, the infimum taken over all finite coverings $\mathscr{U}(\varepsilon)$ of $\mathscr{E}$. Put further $H(\varepsilon)=\log N(\varepsilon)$. Then $H(\varepsilon)$ is a measure of the size of $\varepsilon$-covering required by $\mathscr{E}$, and is called the $\varepsilon$-entropy of $\mathscr{E}$.

Clearly $H(\varepsilon) \uparrow \infty$ as $\varepsilon \downarrow 0$. Here the rate of increase of $H(\varepsilon)$ can be effectively compared with that of $1 / \varepsilon$. Put

$$
\begin{aligned}
\rho(\mathscr{E}) & =\operatorname{glb}\left\{\mu>0: H(\varepsilon) \leqq \text { const }(1 / \varepsilon)^{\mu}\right\} \\
& =\infty \text { if no such } \mu>0 \text { exists. }
\end{aligned}
$$

Then $\rho(\mathscr{E})$ is the order of growth of the $\varepsilon$-entropy of $\mathscr{E}$. The value of $\rho(\mathscr{E})$ can evidently be computed from the formula

$$
\rho(\mathscr{E})=\limsup _{\varepsilon \rightarrow \infty} \frac{\log H(\varepsilon)}{\log 1 / \varepsilon}
$$

when $\mathscr{E}=K(\mathscr{B})$ we write $\rho(\mathscr{E})=\rho(K)$.

Other measures of the "size" of $K$ may be obtained in various other ways. Among them we cite the following:

Definition 7 (The Eigenvalue Sequence). As before, let $k_{n}$ be the $n$th eigenvalue, counted with multiplicities and arranged in order of decreasing magnitude, of the operator $K$. Since $K$ is compact, $\left|k_{n}\right| \downarrow 0$ as $n \uparrow \infty$. The rate of decrease of $\left|k_{n}\right|$ can be effectively compared with that of $1 / n$. Put

$$
\begin{aligned}
\kappa(K) & =\operatorname{lub}\left\{\mu>0:\left|k_{n}\right| \leqq \text { const }(1 / n)^{\mu}\right\} \\
& =0 \text { if no such } \mu>0 \text { exists. }
\end{aligned}
$$

Then $\kappa(K)$ is the order of decay of the eigenvalue sequence of $K$.

Definition 8 (Behavior on ORthonormal Bases). Let $\Phi=\left\{\phi_{n}\right\}$ be any orthonormal basis for $\mathscr{H}$, and put $l_{n}=\left\|K \phi_{n}\right\|$. Assume the $l_{n}$ are arranged in order of decreasing magnitude. Since $K$ is compact, $l_{n} \downarrow 0$ as $n \uparrow \infty$. Put

$$
\begin{aligned}
\lambda(K, \Phi) & =\operatorname{lub}\left\{\mu>0: l_{n} \leqq \text { const }(1 / n)^{\mu}\right\}, \\
& =0 \text { if no such } \mu>0 \text { exists. }
\end{aligned}
$$

Then $\lambda(K, \Phi)$ is the order of decay of the sequence $\left\|K \phi_{n}\right\|$.

Definition 9 (The Fredholm Determinant [2, p. 1106ff]). Now let $K$ be a normal compact operator, and assume $\tau(K)<\infty$. Let $k=[\tau(K)]$ be the greatest integer in $\tau(K)$, and $z$ be any complex number. For each integer $j>k$, put $\sigma_{j}$ $=\operatorname{trace}\left(K^{j}\right)$, and form

$$
d(z, K)=\operatorname{det}_{k}(I-z K)=\exp \left\{-\sum_{j=k+1}^{\infty} \frac{\sigma_{j} z^{j}}{j}\right\}
$$


then $d(z, K)$ is the (generalized) Fredholm determinant of $I-z K$. We know that $d(z, K)$ is a well-defined complex-valued function of $z$, analytic in the whole $z$-plane [2, p. 1106]. Let $M(r, d)$ be the maximum modulus of $d(z, K)$ on the circle $|z|=r$

$$
M(r, d)=\max _{|z|=r}|d(z, K)|
$$

then $M(r, d) \uparrow \infty$ as $r \uparrow \infty$. The rate of growth of $\log M(r, d)$ can be effectively compared with that of $r$. Put

$$
\gamma(K)=\operatorname{glb}\left\{\mu>0: \log M(r, d) \leqq \text { const } r^{\mu}\right\} .
$$

Then $\gamma(K)$ is the exponential order of growth of $d(z, K)$. To compute $\gamma(K)$, we use

$$
\gamma(K)=\limsup _{r \rightarrow \infty} \frac{\log \log M(r, d)}{\log r} .
$$

Now for any $z$ for which $z^{-1}$ lies in the resolvent set of $K$, let $R\left(z^{-1}, K\right)$ $=z(I-z K)^{-1}$ be the resolvent of $K$, and put

$$
D(z, K)=d(z, K) R\left(z^{-1}, K\right)
$$

then $D(z, K)$ is the (generalized) Fredholm minorant of $K$. It is known that $D(z, K)$ is a well-defined operator-valued function of $z$, which admits an analytic extension to the whole $z$-plane [2, p. 1112]. If we define the maximum modulus by

$$
M(r, D)=\max _{|z|=r}\|D(z, K)\|,
$$

then $M(r, D) \uparrow \infty$ as $r \uparrow \infty$. The rate of growth of $\log M(r, D)$ is then given by

$$
\Gamma(K)=\operatorname{glb}\left\{\mu>0: \log M(r, D) \leqq \text { const } r^{\mu}\right\} .
$$

Then $\Gamma(K)$ is the exponential order of growth of $D(z, K)$. To compute $\Gamma(K)$, we use

$$
\Gamma(K)=\limsup _{r \rightarrow \infty} \frac{\log \log M(r, D)}{\log r} .
$$

Definition 10 (The Fredholm Coefficients). Again let $K$ be a normal compact operator and $d(z, K)$ and $D(z, K)$ the entire functions introduced in Definition 9. Write

$$
d(z, K)=\sum_{n=0}^{\infty} d_{n}(K) z^{n}
$$

here $d_{n}(K)$ is the $n$th Taylor coefficient of $d(z, K)$ in the Taylor series expansion about the origin. Since $d_{n}(z, K)$ is entire, we must have $d_{n} \rightarrow 0$ as $n \rightarrow \infty$. The rate of decrease of the $d_{n}$ can be effectively compared with that of $1 / n !$. Put

$$
\delta(K)=\operatorname{lub}\left\{\mu>0:\left|d_{n}(K)\right| \leqq \text { const }(1 / n !)^{\mu}\right\} .
$$


Then $\delta(K)$ is the order of decay of the Fredholm coefficients $d_{n}$ relative to $1 / n !$.

Similarly, we have

$$
D(z, K)=\sum D_{n}(K) z^{n}
$$

where $D_{n}(K)$ is the $n$th Taylor coefficient of $D(z, K)$. Since $D(z, K)$ is entire, we must have $\left\|D_{n}(K)\right\| \rightarrow 0$ as $n \rightarrow \infty$. Now put

$$
\Delta(K)=\operatorname{lub}\left\{\mu>0:\left\|D_{n}(K)\right\| \leqq \text { const }(1 / n !)^{\mu}\right\}
$$

then $\Delta(K)$ is the corresponding order of decay of the $D_{n}(K)$ relative to $1 / n$ !.

Definition 11 (The Resolvent). Again let $K$ be a normal compact operator, and assume $\tau(K)<\infty$. For $z$ any complex number with $z^{-1}$ in the resolvent set of $K$, let $R\left(z^{-1}, K\right)=z(I-z K)^{-1}$. Then $R\left(z^{-1}, K\right)$ is an operator-valued function of $z$, meromorphic in the whole $z$-plane. In fact, we have $R\left(z^{-1}, K\right)=D(z, K) / d(z, K)$.

To estimate the rate of growth of $R\left(z^{-1}, K\right)$ as $|z| \rightarrow \infty$, we must replace the maximum modulus with something a little less sensitive to the presence of poles. For this purpose we introduce the characteristic function of Nevanlinna, defined as follows (cf. [3, p. 4]).

Let $n(r, R)$ denote the number of poles of $R\left(z^{-1}, K\right)$ (i.e., the number of zeros of $d(z, K)$ ) lying inside the circle $|z|=r$, and define (note that $n(0, R)=0$ )

$$
N(r, R)=\int_{0}^{r} n(t, R) \frac{d t}{t} .
$$

Furthermore, for $x>0$, put $\log ^{+} x=\max \{\log x, 0\}$, and define

$$
m(r, R)=\frac{1}{2 \pi} \int_{0}^{2 \pi} \log ^{+}\left\|R\left(r^{-1} e^{-i \theta}, K\right)\right\| d \theta .
$$

Finally, define

$$
T(r, R)=N(r, R)+m(r, R) .
$$

Then $T(r, R)$ is Nevanlinna's characteristic function, designed to play the role of $\log M(r, D)$ for $R$. Clearly $m(r, R)$ is a weighted average of the modulus of $R\left(z^{-1}, K\right)$ on the circle $|z|=r$, and $N(r, R)$ counts the number of poles inside this circle. We shall see that $T(r, R)$ is finite for all $r$, and that $T(r, R) \uparrow \infty$ as $r \uparrow \infty$ (cf. [3, p. 8]). The rate of growth of $T(r, R)$ is measured by

$$
\zeta(K)=\operatorname{glb}\left\{\mu>0: T(r, R) \leqq \text { const } r^{\mu}\right\} .
$$

Clearly we have

$$
\zeta(K)=\limsup _{r \rightarrow \infty} \frac{\log T(r, R)}{\log r} .
$$

This completes our enumeration of possible measures of the "size" of $K$. We now propose to show that they are essentially all the same. 
THEOREM 12. Let $K$ be any compact operator acting on $\mathscr{H}$, and $A=\left(K^{*} K\right)^{1 / 2}$. Assume $\tau(K)<\infty$. Then (see Definitions 4-11)

$$
\begin{gathered}
2 /(\beta(K)-1)=1 / \omega(K)=\rho(K)=\tau(K), \\
\gamma(A)=\Gamma(A)=1 / \delta(A)=1 / \Delta(A)=\zeta(A)=1 / \kappa(A)=\tau(A)=\tau(K) .
\end{gathered}
$$

If $K$ is normal, then

$$
\gamma(K)=\Gamma(K)=1 / \delta(K)=1 / \Delta(K)=\zeta(K)=1 / \kappa(K)=\tau(K) .
$$

If $2 \leqq \tau(K)<\infty$, then $\lambda(K, \Phi)=\lambda(K)$ is independent of $\Phi$, and

$$
\lambda(K)=\tau(K) .
$$

The proof of (40) depends on the following observations: Let $\left\{k_{n}\right\}$ be the eigenvalue sequence of $K$, counted with multiplicities and arranged in order of decreasing magnitude. For each $\varepsilon>0$, let $n(\varepsilon)=\max \left\{n:\left|k_{n}\right| \geqq \varepsilon\right\}$. Define

$$
\begin{aligned}
& \kappa_{1}(K)=\operatorname{lub}\left\{\mu:\left|k_{n}\right| \leqq \text { const }(1 / n)^{\mu}\right\} \\
& \kappa_{2}(K)=\operatorname{lub}\left\{\mu:\left|\prod_{i=1}^{n} k_{i}\right| \leqq \text { const }(1 / n !)^{\mu}\right\} \\
& \tau_{1}(K)=\operatorname{glb}\left\{\mu: n(\varepsilon) \leqq \text { const }(1 / \varepsilon)^{\mu}\right\} \\
& \tau_{2}(K)=\operatorname{glb}\left\{\mu: \sum\left|k_{n}\right|^{\mu}<\infty\right\}
\end{aligned}
$$

LEMMA 13. With $\kappa_{1}, \kappa_{2}$ and $\tau_{1}, \tau_{2}$ as defined in (44), we have

$$
\tau_{1}=\tau_{2}=1 / \kappa_{1}=1 / \kappa_{2} \text {. }
$$

Proof. That $\tau_{1}=\tau_{2}$ is classic, and is proved e.g. in $\left[4\right.$, p. 10]. That $\tau_{1}=1 / \kappa_{1}$ is proved as follows (cf. [1]):

For any $\mu>\kappa_{1}$, we have $\left|k_{n}\right| \leqq$ const $(1 / n)^{\mu}$ for all $n$. Now given $\varepsilon>0$, choose $n=n(\varepsilon)$, and note $\varepsilon \leqq\left|k_{n(\varepsilon)}\right| \leqq$ const $(1 / n(\varepsilon))^{\mu}$. Hence $n(\varepsilon) \leqq(\text { const } / \varepsilon)^{1 / \mu}$ for all $\varepsilon>0$, and so $\tau_{1} \leqq 1 / \mu$. Conversely, if $\mu<\kappa_{1}$, we have $\left|k_{n}\right| \geqq$ const $(1 / n)^{\mu}$ for arbitrarily large $n$. Given such an $n$, choose $\varepsilon=\left|k_{n}\right|$, and note $\varepsilon=\left|k_{n}\right| \geqq$ const $(1 / n(\varepsilon))^{\mu}$. Hence $n(\varepsilon) \leqq(\text { const } / \varepsilon)^{1 / \mu}$ for arbitrarily small $\varepsilon$, and so $\tau_{1} \geqq 1 / \mu$. Since $\mu$ is arbitrary, we have proved $\tau_{1} \leqq 1 / \kappa_{1} \leqq \tau_{1}$.

To show that $\kappa_{1}=\kappa_{2}$, note first that for any $\mu<\kappa_{1},\left|k_{n}\right| \leqq$ const $(1 / n)^{\mu}$ for all $n$. Hence $\left|\prod_{i=1}^{n} k_{i}\right| \leqq(\text { const })^{n}(1 / n !)^{\mu} \leqq$ const $(1 / n !)^{\nu}$ for any $\nu<\mu$. Hence $\kappa_{1} \leqq \kappa_{2}$. Similarly, for any $\mu>\kappa_{1},\left|k_{n}\right| \geqq$ const $(1 / n)^{\mu}$ for arbitrarily large $n$, and so $\left|\prod_{i=1}^{n} k_{i}\right|$ $\geqq(\text { const })^{n}(1 / n)^{n \mu} \geqq$ const $(1 / n !)^{\nu}$ for any $\nu>\mu$. Hence $\kappa_{2} \leqq \kappa_{1}$, and so $\kappa_{1}=\kappa_{2}$.

To prove $(40)$ we now simply observe that the image $\mathscr{E}=K(\mathscr{B})$ of the unit ball $\mathscr{B}$ under $K$ is a compact ellipsoid, whose semiaxes are just the eigenvalues $a_{n}$ of $A$ 
(see [6]). It then follows directly that the $n$-dimensional metric volume $V_{n}$ is given by

$$
V_{n}=B_{n} \prod_{i=1}^{n} a_{i}
$$

while the $n$-dimensional metric width $w_{n}$ is given by

Hence

$$
w_{n}=a_{n} \text {. }
$$

$$
\begin{aligned}
\beta(K) & =\liminf _{n \rightarrow \infty} \frac{\log V_{n}}{\log B_{n}} \\
& =\liminf _{n \rightarrow \infty} \frac{2 \log \prod_{i=1}^{n} a_{i}}{\log (1 / n !)}+1 \\
& =2 \kappa_{2}(A)+1,
\end{aligned}
$$

so $(\beta(K)-1) / 2=\kappa_{2}(A)$. Here we have used the known fact that

$$
\lim _{n \rightarrow \infty}\left(\log B_{n} / \log 1 / n !\right)=1 / 2 \text {. }
$$

Since $w_{n}=a_{n}$ for all $n$, we have $\omega(K)=\kappa_{1}(A)$.

Furthermore, we know that the number $N(\varepsilon)$ of elements in an optimal $\varepsilon$-covering of $\mathscr{E}$ is bounded above and below by (see [6])

$$
\prod_{i=1}^{n(2 \varepsilon)} \frac{a_{i}}{\varepsilon} \leqq N(\varepsilon) \leqq \prod_{i=1}^{n(\varepsilon / 4 \sqrt{ } 2)} \frac{4 \sqrt{ } 2 a_{i}}{\varepsilon}
$$

Since $a_{1} \geqq a_{2} \geqq \cdots \geqq a_{n} \geqq 0$, we have

$$
2^{n(2 \varepsilon)} \leqq N(\varepsilon) \leqq(4 \sqrt{ } 2 / \varepsilon)^{n(\varepsilon / 4 \sqrt{ } 2)} .
$$

Hence

$$
n(2 \varepsilon) \log 2 \leqq H(\varepsilon) \leqq n(\varepsilon / 4 \sqrt{ } 2) \log (4 \sqrt{ } 2 / \varepsilon) .
$$

By dividing through by $\log (1 / \varepsilon)$ and taking the limit supremum as $\varepsilon \rightarrow 0$, we get

$$
\tau_{1}(A) \leqq \beta(K) \leqq \tau_{1}(A) \text {. }
$$

From Lemma 13 we have $1 / \kappa_{1}(A)=1 / \kappa_{2}(A)=\tau_{1}(A)=\tau_{2}(A)=\tau(A)=\tau(K)$. Hence

$$
2 /(\beta(K)-1)=1 / \omega(K)=\beta(K)=1 / \kappa(A)=\tau(A)=\tau(K) .
$$

Moreover, if $K$ is normal, then clearly $\left|k_{n}\right|=a_{n}$, and so $\kappa(K)=\kappa(A)$.

The proof of (41) depends on the following result: Let $f(z)$ be an entire function of $z$, of finite genus. Let $d_{n}$ be the $n$th coefficient of the Taylor series for $f$ computed at the origin, and let $z_{n}$ be the $n$th zero of $f$, counted with multiplicities and arranged in order of increasing magnitude. Define

$$
\begin{aligned}
& \delta=\operatorname{lub}\left\{\mu:\left|d_{n}\right| \leqq \text { const }(1 / n !)^{\mu}\right\}, \\
& \gamma=\operatorname{glb}\left\{\mu:|f(z)| \leqq \exp \text { const }|z|^{\mu}\right\}, \\
& \tau=\operatorname{glb}\left\{\mu: \sum\left|z_{n}\right|^{-\mu}<\infty\right\} .
\end{aligned}
$$


LEMMA 14. With $\gamma, \delta$ and $\tau$ as defined above, we have

$$
1 / \delta=\gamma=\tau \text {. }
$$

Proof. The fact that $\gamma=\tau=\lim \sup _{n \rightarrow \infty}\left(n \log n / \log 1 /\left|d_{n}\right|\right)$ is classic (see[4, Chapter 1]). Here we need only observe that

$$
\delta=\liminf _{n \rightarrow \infty} \frac{\log \left|d_{n}\right|}{\log (1 / n !)}
$$

Hence

$$
1 / \delta=\limsup _{n \rightarrow \infty} \frac{\log n !}{\log 1 /\left|d_{n}\right|}=\gamma=\tau .
$$

To prove (41), we observe that, if $K$ is normal, and $\tau(K)<\infty$, then $d(z, K)$ $=\operatorname{det}_{k}(I-z K)$ is given by

$$
\begin{aligned}
d(z, K) & =\exp \operatorname{tr}\left\{-\sum_{j=k+1}^{\infty} \frac{(z K)^{j}}{j}\right\} \\
& =\prod_{n=1}^{\infty}\left\{\left(1-z k_{n}\right) \exp \sum_{j=1}^{k} \frac{\left(z k_{n}\right)^{j}}{j}\right\}
\end{aligned}
$$

(see [2, p. 1106]). Hence $d(z, K)$ is an entire function of $z$, of finite genus, whose zeros are $z_{n}=1 / k_{n}$, and whose Taylor coefficients are $d_{n}(K)$. It follows immediately from Lemma 14 that

$$
1 / \delta(K)=\gamma(K)=\tau(K) .
$$

If now $K$ is arbitrary, then $A$ is normal, and

$$
1 / \delta(A)=\gamma(A)=\tau(A)=\tau(K) .
$$

A similar argument holds for $D(z, K)$. Assume $K$ is normal, and $\tau(K)<\infty$. Then for any eigenvalue $k_{n}$ of $K$ and any $z$ with $|z|>1$ we have

$$
\left|z /\left(1-z k_{n}\right)\right| \geqq 1 /\left(1+\left|k_{n}\right|\right) \geqq 1 /\left(1+\left|k_{1}\right|\right) \text {. }
$$

It follows that the resolvent $R\left(z^{-1}, K\right)$ of $K$ satisfies

$$
\left\|R\left(z^{-1}, K\right)\right\| \geqq 1 /(1+\|K\|)
$$

for all $z$ with $|z|>1$. Hence $D(z, K)=d(z, K) R\left(z^{-1}, K\right)$ satisfies

$$
\|D(z, K)\| \geqq|d(z, K)| /(1+\|K\|)
$$

for all $z$ with $|z|>1$.

It follows that

$$
\Gamma(K) \geqq \gamma(K) .
$$

On the other hand, if $\mu>\tau(K)$, then we know that

$$
\|D(z, K)\| \leqq|z| \exp \left\{\text { const }|z|^{\mu}\|K\|_{\mu}^{\mu}\right\}
$$

(see [2, p. 1112]). Hence $\Gamma(K) \leqq \mu$, and so $\Gamma(K) \leqq \tau(K)=\gamma(K)$. 
The proof that $\Delta(K)=1 / \Gamma(K)$ is the operator analogue of the proof that $\delta(K)$ $=1 / \gamma(K)$, and will not be presented here (see $[4$, p. 4]).

Thus when $K$ is normal, we have $1 / \Delta(K)=\Gamma(K)=\tau(K)$. When $K$ is arbitrary, we have $1 / \Delta(A)=\Gamma(A)=\tau(A)=\tau(K)$.

We note in passing that the order of decay of the Fredholm coefficients is of some interest in the problem of computing approximants to $R\left(z^{-1}, K\right)=$ $D(z, K) / d(z, K)$. The asymptotic accuracy of the approximants can be estimated from the values of $\delta(K)$ and $\Delta(K)$, which in turn can be determined from the value of the invariant $\tau(K)$.

For the resolvent, we argue as follows: With $N(r, R), m(r, R)$ and $T(r, R)$ defined as in (35), (36) and (37), note that $n(r, R)$ is the number of poles of $R\left(z^{-1}, K\right)$ inside $|z|=r$, i.e., the number of zeros of $d(z, K)$ inside $|z|=r$, which is just the number of eigenvalues $k_{n}$ of $K$ with $\left|k_{n}\right| \geqq 1 / r$. By Lemma 13 , then, $n(r, R)$ has order of growth $\tau(K)$. It follows that

$$
N(r, R)=\int_{0}^{r} n(t, R) \frac{d t}{t}
$$

also has order of growth $\tau(K)$.

To compute the order of growth of $m(r, R)$, first note that

$$
\left\|R\left(z^{-1}, K\right)\right\|=\left|d(z, K)^{-1}\right|\|D(z, K)\| .
$$

Hence

$$
\log ^{+}\left\|R\left(z^{-1}, K\right)\right\| \leqq \log ^{+}\left|d(z, K)^{-1}\right|+\log ^{+}\|D(z, K)\| .
$$

Thus $m(r, R) \leqq m(r, 1 / d)+m(r, D)$.

Now $m(r, D) \leqq \log ^{+} M(r, D)=\log M(r, D)$ for $r$ sufficiently large. Here $M(r, D)$ is the maximum modulus of $D(z, K)$. The order of growth $\Gamma(K)$ of $\log M(r, D)$ we have shown to be equal to $\tau(K)$.

For $m(r, 1 / d)$, we observe that from Jensen's Theorem we have (cf. [3, p. 4])

$$
m(r, 1 / d)+N(r, 1 / d)=m(r, d)+N(r, d) .
$$

But since $d(z, K)$ is entire, $N(r, d)=0$. Moreover, for large $r, m(r, d) \leqq \log M(r, d)$, whose order of growth $\gamma(K)$ is equal to $\tau(K)$. Finally $N(r, 1 / d)=N(r, R)$ has order of growth $\tau(K)$, as shown above. It follows that $m(r, 1 / d)$ has order of growth at most $\tau(K)$.

Hence $T(r, R)=m(r, R)+N(r, R)$ has order of growth equal to the maximum of that of $m(r, R)$ and $N(r, R)$, which is just $\tau(K)$, as required.

We have shown that if $K$ is normal, then $\zeta(K)=\tau(K)$. When $K$ is arbitrary, then $A$ is normal, and we have $\zeta(A)=\tau(A)$.

It remains to prove (43). Let $K$ be any compact operator with $2 \leqq \tau(K)<\infty$, and $\Phi=\left\{\phi_{n}\right\}$ any orthonormal basis for $\mathscr{E}$. We know that if $2 \leqq \lambda(K, \Phi)<\mu$, then 
$\sum\left\|K \phi_{n}\right\|^{\mu}<\infty$ and so $\|K\|_{\mu}<\infty$ (cf. $[2$, p. 1106]) and so $\lambda(K)<\mu$. Hence $\lambda(K)$ $\leqq \lambda(K, \Phi)$.

Conversely, if $2 \leqq \tau(K)<\mu$, then we know that $\|K\|_{\mu}<\infty$, and so

$$
\sum\left\|K \phi_{n}\right\|^{\mu}=\sum\left(A^{2} \phi_{n}, \phi_{n}\right)^{\mu / 2}<\text { const }\left(\left\|A^{2}\right\|_{\mu / 2}\right)=\text { const }\left(\|K\|_{\mu}\right)^{\mu}<\infty
$$

(cf. [2, p. 1138]). Thus $\lambda(K, \Phi)<\mu$, and so $\lambda(K, \Phi) \leqq \tau(K)$.

Note that this result is independent of the choice of basis $\Phi$.

The argument also proves that if $\tau(K) \leqq 2$, then $\lambda(K, \Phi) \leqq 2$, and if $\lambda(K, \Phi) \leqq 2$ then $\tau(K) \leqq 2$. In these cases, however, $\lambda(K, \Phi)$ is no longer independent of the basis $\Phi$ and equals $\tau(K)$ only for bases "sufficiently close" to the eigenfunctions of $A$.

\section{BIBLIOGRAPHY}

1. R. M. Dudley, The sizes of compact subsets of Hilbert space and continuity of Gaussian processes (to appear).

2. N. Dunford and J. T. Schwartz, Linear operators. Part II: Spectral theory, Interscience, New York, 1963.

3. W. K. Hayman, Meromorphic functions, Clarendon, Oxford, 1964.

4. B. Ja. Levin, Distribution of zeros of entire functions, Transl. Math. Monographs, Vol. 5, Amer. Math. Soc., Providence, R. I., 1964.

5. G. G. Lorentz, Metric entropy, widths, and superpositions of functions, Amer. Math. Monthly 69 (1962), 469-485.

6. R. T. Prosser and W. L. Root, The e-entropy and e-capacity of certain time-invariant channels, J. Math. Anal. Appl. 21 (1968), 233-241.

\section{Dartmouth College,}

HANOVER, New HAMPSHIRE 